JMB

\title{
Rapid Determination of Protein Folds Using Residual Dipolar Couplings
}

\section{Andrew Fowler ${ }^{1,2}$, Fang Tian', Hashim M. Al-Hashimi ${ }^{1,2}$ and James H. Prestegard ${ }^{1 *}$}

${ }^{1}$ Complex Carbohydrate

Research Center, The

University of Georgia, 220

Riverbend Road, Athens

GA 30602-4712, USA

${ }^{2}$ Department of Chemistry, Yale University, PO Box 208107

New Haven, CT 06520-

8107, USA

\begin{abstract}
Over the next few years, various genome projects will sequence many new genes and yield many new gene products. Many of these products will have no known function and little, if any, sequence homology to existing proteins. There is reason to believe that a rapid determination of a protein fold, even at low resolution, can aid in the identification of function and expedite the determination of structure at higher resolution. Recently devised NMR methods of measuring residual dipolar couplings provide one route to the determination of a fold. They do this by allowing the alignment of previously identified secondary structural elements with respect to each other. When combined with constraints involving loops connecting elements or other short-range experimental distance information, a fold is produced. We illustrate this approach to protein fold determination on ${ }^{15} \mathrm{~N}$-labeled Eschericia coli acyl carrier protein using a limited set of ${ }^{15} \mathrm{~N}-{ }^{1} \mathrm{H}$ and ${ }^{1} \mathrm{H}-{ }^{1} \mathrm{H}$ dipolar couplings. We also illustrate an approach using a more extended set of heteronuclear couplings on a related protein, ${ }^{13} \mathrm{C}_{1}^{15} \mathrm{~N}$-labeled NodF protein from Rhizobium leguminosarum.
\end{abstract}

(C) 2000 Academic Press

Keywords: structural genomics; NMR; ACP; NodF; protein structure

\section{Introduction}

Current efforts directed toward sequencing the complete genomes of various species are producing results at an astonishing rate. Many of the identified genes code for proteins with no known function, and have a low level of sequence homology to previously characterized proteins. ${ }^{1,2}$ Structural genomics provides a route to functional characterization by firstly obtaining the three dimensional structures of large numbers of representative proteins. ${ }^{1,3,4}$ Various computational methods would then be used to predict specific activities or other properties of biological

Present address: H. M. Al-Hashimi, Cellular Biochemistry and Biophysics Program, Memorial SloanKettering Cancer Center, New York, NY 10021, USA.

Abbreviations used: NOE, nuclear Overhauser effect; ACP, acyl carrier protein; FFFs, fuzzy functional forms; IPTG, isopropyl $\beta$-D-thiogalactopyranoside; SDS-PAGE, sodium dodecyl sulfate - polyacrylamide gel electrophoresis; HPLC, high performance liquid chromatography; LB, Luria-Bertani broth; POSE, positioning of structural elements.

E-mail address of the corresponding author: jpresteg@ccrc.uga.edu interest. ${ }^{5-9}$ The challenge arises in the shear number of structure determinations which must be undertaken. Here we discuss one approach that can help meet this challenge.

Nuclear magnetic resonance spectroscopy (NMR) provides a means of obtaining structural information that complements X-ray crystallography by being applicable in solution to proteins that may be resistant to a crystallographic approach. Traditional NMR techniques for the determination of macromolecular structure, however, are not well suited to genomics applications because of the vast amount of time required to produce a structure. These methods rely heavily upon refinement against distance constraints obtained from NOE measurements. Because of the steep distance dependence $\left(1 / r^{6}\right)$, and resultant short-range character of NOEs, critical fold determining constraints often come from side-chain to side-chain contacts in the hydrophobic core of proteins. This results in the requirement that the vast majority of all observed resonances in the NMR spectrum are assigned, including those at the very extremes of amino acid side-chains. Much of the time involved in determining a structure is consumed during the resonance assignment and NOE identification 
stage. Software is currently available that can at least partially automate these stages; ${ }^{10-16}$ however, the minimum set of experiments that must be performed in order for these routines to work reliably can often require well over a month of data acquisition. With the number of samples expected in any sort of high throughput genomics application, a more rapid means of extracting structural information is required.

Recently developed NMR methodology based on the measurement of residual dipolar couplings in partially ordered media can provide an alternative means of rapidly obtaining structural information. ${ }^{17,}{ }^{18}$ Residual dipolar couplings complement NOE data in that they provide angular restraints that can be used to orient remote structural elements in a protein, even when the intervening distance is too great to allow a determination using NOE-based methods. ${ }^{19-22}$ One can think of any reasonably rigid piece of a protein as a structural element; in particular, regular elements of secondary structure, such as $\alpha$ or $3_{10}$-helices, $\beta$-sheets, and reverse turns, can serve as these elements. The identification of these elements can be done relatively easily from short and intermediate-range NOEs involving backbone protons, or directly from the experiments needed to make sequential backbone resonance assignments. ${ }^{23,24}$ Once the secondary structural elements are identified and backbone resonance assignments are made, residual dipolar couplings adequate for fold determination can be measured using backbone resonances alone. Here, we focus entirely on those residual dipolar couplings which exist between amide protons and directly bonded amide nitrogen atoms $\left({ }^{1} \mathrm{D}_{\mathrm{NH}}\right)$, alpha carbon atoms and directly bonded alpha protons $\left({ }^{1} \mathrm{D}_{\mathrm{CH}}\right)$, intra or inter-residue proton-proton pairs involving amide and alpha protons $\left(\mathrm{D}_{\mathrm{HH}}\right)$, and a given carbonyl carbon atom and the amide nitrogen atom or proton of a sequential residue $\left({ }^{1} \mathrm{D}_{\mathrm{NC}^{\prime}}{ }^{2} \mathrm{D}_{\mathrm{HC}}\right)$.

The magnitude of an observed dipolar coupling is given by:

$$
D_{i j}=\frac{-\mu_{0} \gamma_{i} \gamma_{j} \hbar}{4 \pi^{2} r^{3}}\left\langle\frac{3 \cos ^{2} \theta-1}{2}\right\rangle
$$

where $\gamma_{i}$ and $\gamma_{j}$ are the gyromagnetic ratios of the $i$ th and $j$ th nuclei, $r$ is the distance between nuclei $i$ and $j$, and $\theta_{i}$ is the angle between the internuclear vector and the magnetic field (see Figure 1). The angle brackets denote a time average; in isotropic solution the angular term averages to zero and dipolar couplings are not observed, but if the sample is partially oriented we can measure a residual dipolar coupling which manifests itself in a manner similar to scalar couplings. Provided the measurement of at least five independent residual dipolar couplings per structural element can be can be made, the preferred orientation of all elements can be determined.

An order matrix approach proves convenient for determination of element orientation. ${ }^{25}$ The angular

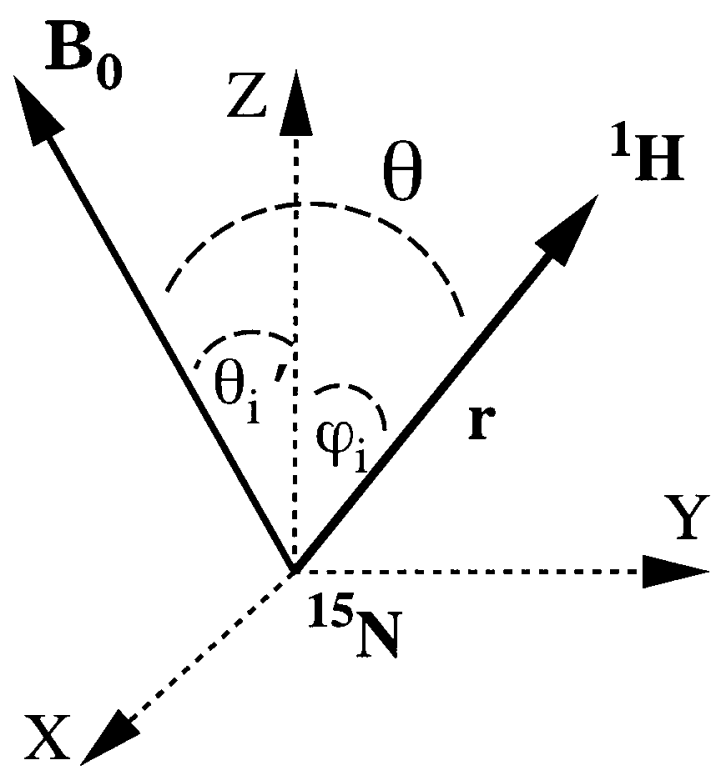

Figure 1. Diagram illustrating the parameters which are required to define a dipolar coupling and the elements of the Saupe order matrix.

term in equation (1) can be rewritten as:

$$
\left\langle\frac{3 \cos ^{2} \theta_{k}-1}{2}\right\rangle=\sum_{i, j=\{x, y, z\}} S_{i j} \cos \phi_{i k} \cos \phi_{j k}
$$

where $\phi_{i k}$ is the angle between the $k$ th internuclear vector and the $i$ th axis of an arbitrarily chosen coordinate frame and $S_{i j}$ are Saupe order parameters. These are given by:

$$
S_{i j}=\left\langle\frac{3 \cos ^{2} \theta_{i}^{\prime} \cos ^{2} \theta_{j}^{\prime}-\delta_{i j}}{2}\right\rangle
$$

where $\theta_{i}^{\prime}$ is the angle between the $i$ th axis of our arbitrarily chosen frame and the magnetic field, and $\delta_{i j}$ is the Kronecker delta function (Figure 1).

The order matrix parameters, only five of which are independent, can be determined from a set of measured couplings and the assembled order matrix diagonalized to give three Euler angles relating a secondary structure element to its principal alignment frame and two principal order parameters describing the extent and asymmetry of order $\left(S_{\mathrm{zz}}\right.$ and $\eta=\left(S_{\mathrm{yy}}-S_{\mathrm{xx}}\right) / S_{\mathrm{zz}}$, respectively). For a rigid molecule, all structural elements must see the same molecular alignment frame; thus, we can orient all rigid elements of a molecule correctly with respect to each other by superimposing their alignment frames. Subsequent translation of elements to satisfy a minimum set of distance constraints produces a backbone structure, or protein fold.

We demonstrate the above approach on ${ }^{15} \mathrm{~N}$ labeled Escherichia coli acyl carrier protein (ACP), a 
77-amino acid protein that carries a phosphopantetheine prosthetic group at Ser36. ACP is one of the most abundant soluble proteins in E. coli, shuttling growing acyl chains during fatty acid biosynthesis, as well as serving as an acyl chain donor for a variety of enzymes and cellular processes. ${ }^{26,27}$ We also illustrate a variation of our method which makes use of a much larger number of dipolar couplings on ${ }^{13} \mathrm{C},{ }^{15} \mathrm{~N}$ doubly labeled NodF protein from Rhizobium leguminosarum, a 92-amino acid protein. NodF, combined with the NodE gene product, is required for the biosynthesis of hostspecific, polyunsaturated fatty acids which are used in the production of lipochitin oligosaccharide signals for symbiotic root nodulation on host plants. ${ }^{28,29}$ NodF also has a relatively high level of homology with ACP, especially around the prosthetic group attachment site (S36 in ACP, S45 in NodF), and, in fact, chimeras of the two proteins which retain acyl donor activity have been produced. ${ }^{30}$ Thus, structural comparison of these two proteins is of some interest.

\section{Results}

For ACP, both sequential and NOE assignments have been previously reported based upon both homonuclear and heteronuclear experiments. ${ }^{31-33}$ In a test of an ability to make assignments and identify secondary structural elements using a minimum of data sets, we examined ${ }^{15} \mathrm{~N}-\mathrm{NOESY}$ HSQC $^{34}$ and HNHA ${ }^{35}$ scalar coupling data sets. Only ${ }^{1} \mathrm{H}_{-}{ }^{15} \mathrm{~N}$ amide pairs that could be identified as being sequentially connected and belonging to a particular type of secondary structure ( $\alpha$-helix) were used in subsequent analyses. In general, the appearance of strong $\mathrm{H}^{\mathrm{N}}-\mathrm{H}^{\mathrm{N}}$ connectivities in combination with $i$ to $i+3$ and $i$ to $i+4 \mathrm{H}_{\alpha}-\mathrm{H}^{N}$ correlations or a small value of ${ }^{3} J_{\mathrm{HNHA}}$ were deemed adequate for this conclusion. Connected $\mathrm{H}^{\mathrm{N}}$ resonances were assigned to sequence specific positions based on restriction to particular amino acid types using previously reported RELAYED-COSY data. ${ }^{31}$ In a modern application the RELAYED-COSY data would be replaced with data from a ${ }^{15} \mathrm{~N}$-TOCSYHSQC experiment. As an example of the proceedure, a stretch of five connected amino acid residues Gly-X-X-X-Gly was unambiguously assigned to residues 12 through 16 of ACP, as this is the only stretch of the primary sequence which has two glycine residues separated by three intervening amino acid residues. Similarly, the two consecutive alanine residues at residues 67 and 68 in helix 3 are unique in the primary sequence. The three helices identified and used for the final fold determination ran from Ile3 through Glu13, Leu37 through Asp51, and Val65 through His75. Except for truncation of the first helix by two residues, these correspond to helices identified in the original structural analysis. ${ }^{32,36}$

For NodF, initial assignments and identification of secondary structural elements had been pre- viously reported using ${ }^{15} \mathrm{~N}$-edited NOESY, TOCSY, and HNHA experiments. ${ }^{37,38}$ This actually corresponds to a modern minimal set of experiments for a ${ }^{15} \mathrm{~N}$-labeled protein, and only these experiments were used in the initial backbone assignment. Total data acquisition time was approximately five days on a $4.5 \mathrm{mM}$ sample using a $500 \mathrm{MHz}$ spectrometer for this original set of experiments. Because we had a doubly ${ }^{13} \mathrm{C},{ }^{15} \mathrm{~N}$ labeled sample of NodF and wished to illustrate our approach using a broader range of dipolar couplings, we also assigned the backbone resonances using standard triple resonance methods. ${ }^{39,40}$ HNCACB, $\mathrm{CBCA}(\mathrm{CO}) \mathrm{HN}$, and $\mathrm{HNCO}$ experiments were run on a $2.5 \mathrm{mM}$ sample at $600 \mathrm{MHz}$, requiring a total of roughly two days of instrument time in this recent application. A small number of inconsistencies were found when compared with the original assignments, nearly all of which were at the termini of the protein or in extended loop regions. The identification of helical regions was unaffected and these are used as rigid structural elements for the determination of a fold. The complete set of corrected backbone resonance assignments is given in Table S1 of Supplementary Material. The helices in NodF used for fold determination ran from Leu5 through Val17, Leu46 through Leu58, and Asn76 through Gly86.

Spectra containing dipolar coupling information were obtained for both ACP and NodF in phagecontaining media. ${ }^{41}$ Details of the spectral acquisition and the extraction of dipolar couplings are given in Materials and Methods. Examples of some of the spectra recorded can be found in Supplementary Material. The residual dipolar couplings used in the orientation of helical segments of ACP and NodF, along with those NOEs subsequently used in fold determination, are listed in Tables 1 and 2, respectively. Errors in the measurement of dipolar couplings are approximately $\pm 0.5 \mathrm{~Hz}$ for ${ }^{1} \mathrm{D}_{\mathrm{HN}}$ and ${ }^{1} \mathrm{D}_{\mathrm{CH}}$ couplings measured using phase encoded experiments, and $\pm 1.0 \mathrm{~Hz}$ for all other couplings. Larger errors were used in the determination of the molecular alignment frame, $2-3 \mathrm{~Hz}$ for ${ }^{1} \mathrm{D}_{\mathrm{NH}}$ and correspondingly larger errors for other couplings $\left({ }^{1} \mathrm{D}_{\mathrm{CH}}\right.$ of $3-5 \mathrm{~Hz}, \mathrm{D}_{\mathrm{HH}}$ of 4-6 Hz), mainly to account for deviations between the actual structure of the molecular fragments and our assumption of an idealized rigid geometry.

Analysis of dipolar couplings in terms of a structure was pursued using an order matrix-based program, Orderten_SVD. ${ }^{20}$ The program finds a set of allowable solutions (typically 20,000, requiring less than one minute) by selecting couplings from a normal distribution about the input values, solving a set of equations as given in equations (1) and (2), generating and diagonalizing an order matrix, and keeping any solutions consistent with the original data. Solving the set of linear equations resulting from equations (1) and (2) also requires knowledge of a local structural element so that angles $\phi_{i}$ can be calculated. Local structures used were idealized 
Table 1. Data used to determine the backbone fold of ACP

\begin{tabular}{|c|c|c|c|c|c|c|}
\hline & & & $\mathrm{Cor}$ & & & \\
\hline A. Dipolar couplings in $\alpha$ - & & & & & & \\
\hline Amide couplings & I3 & 1.4 & L37 & -2.6 & Q66 & 8.2 \\
\hline$\left(\mathrm{N}_{i}-\mathrm{H}_{i}^{\mathrm{N}}\right)$ & $\mathrm{E} 4$ & 0.4 & D38 & 1.6 & A67 & 7.7 \\
\hline & E5 & 3.4 & T39 & -0.3 & I69 & 6.8 \\
\hline & V7 & -1.0 & V40 & -2.6 & D70 & 6.1 \\
\hline & K8 & 0.8 & & & N73 & 7.4 \\
\hline & I10 & 2.0 & & & G74 & 5.5 \\
\hline & I11 & -0.3 & & & H75 & 7.7 \\
\hline & G12 & 2.1 & & & & \\
\hline & E13 & 2.1 & & & & \\
\hline & Q14 & 0.0 & & & & \\
\hline & L15 & -1.9 & & & & \\
\hline Amide-alpha couplings & R6 & 3.0 & L37 & 0.4 & Q66 & 0.0 \\
\hline$\left(\mathrm{H}_{i}^{\mathrm{N}}-\mathrm{H}_{\alpha i}\right)$ & V7 & -3.5 & L42 & 0.0 & Ā68 & -8.5 \\
\hline & K9 & 4.5 & V43 & 3.5 & H75 & -9.0 \\
\hline & L15 & 0.0 & M44 & 2.0 & & \\
\hline & & & L46 & -2.0 & & \\
\hline$\left(\mathrm{H}_{i}^{\mathrm{N}}-\mathrm{H}_{\alpha i \pm 1}\right)$ & & & $\mathrm{V} 43_{N}-\mathrm{L} 42_{\alpha}$ & 2.0 & & \\
\hline & & & $\mathrm{M} 44_{\mathrm{N}}-\mathrm{A} 45_{\alpha}$ & -2.0 & & \\
\hline Amide-amide couplings & & & D38 & 2.0 & N73 & 2.0 \\
\hline$\left(\mathrm{H}_{i}^{\mathrm{N}}-\mathrm{H}_{i+1}^{\mathrm{N}}\right)$ & & & T39 & 2.0 & & \\
\hline & & & V40 & 2.0 & & \\
\hline $\begin{array}{l}\text { B. NOEs and distances use } \\
\text { I3 } \mathrm{H}^{\mathrm{N}}-\mathrm{F} 50 \mathrm{H}^{\alpha}, \mathrm{V7} \mathrm{H}^{\mathrm{N}}-\mathrm{F}\end{array}$ & $\begin{array}{l}\text { sition } h \\
l 7 \mathrm{H}^{\alpha}-\mathrm{F}\end{array}$ & $\mathrm{A} 68$ & methyl- & & & \\
\hline $\begin{array}{cccc}6 \AA & 8\end{array}$ & 8 & $\begin{array}{l}1-100 \\
\AA\end{array}$ & $\begin{array}{r}\text { metnyl-1 } \\
8 \stackrel{+}{I}\end{array}$ & & & \\
\hline
\end{tabular}

polyalanine $\alpha$-helices generated using standard backbone torsional angles. ${ }^{42}$

The set of allowable solutions coming from Orderten-SVD is plotted as a distribution of points on Sauson-Flamsteed plots, as shown for the ACP data in Figure 2. The red, black, and blue spots depict the direction of the axes $(z, y$, and $x$, respectively) of the principle alignment frame as seen from each helical element. The spread of solutions gives an approximate picture of the precision with which the alignment frame can be determined. Since dipolar coupling data from a single aligning medium cannot detect inversion of axes, two clusters of solutions appear $180^{\circ}$ apart for each of the axes. This is commonly known as the inversion problem; ${ }^{43}$ four order frames which differ only in the signs of pairs of axes are allowed. The correct frame can be selected either by using a second aligning medium, ${ }^{43}$ or by seeking consistency with other experimental distance information. Since the same set of coordinates was used as input into Orderten-SVD for each helix, the Sauson-Flamsteed projection of the order tensor frame for each helix qualitatively depicts the relative orientation of each

\section{Helix 1}

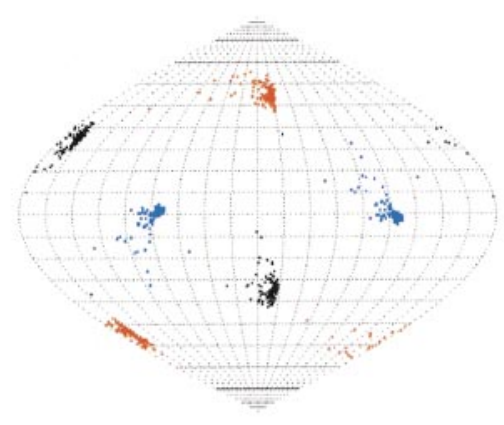

Helix 2

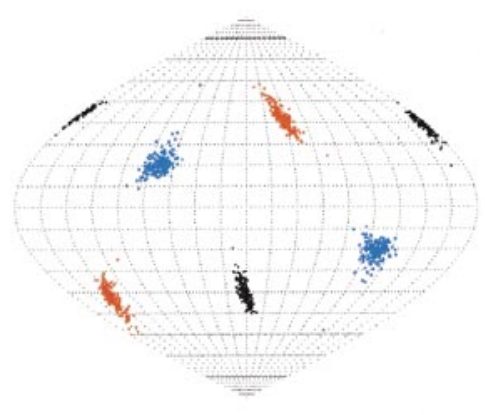

Helix 3

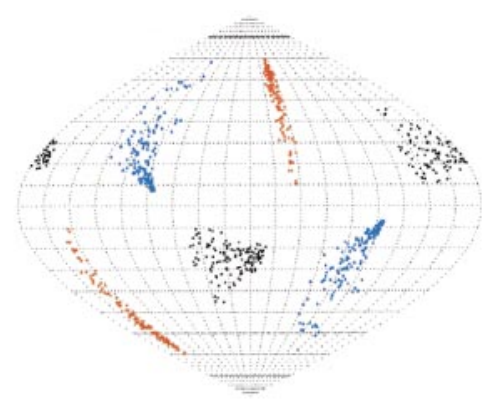

$$
\text { Red }=\text { Szz; } \text { Black }=\text { Syy } ; \text { Blue }=\text { Sxx }
$$

Figure 2. Output from the Orderten-SVD program for the helices of ACP. The various spots correspond to the axes of the principle axis system which is defined by the molecular alignment frame for each helix and are displayed as Sauson-Flamsteed projections. 
Table 2. Data used to determine the backbone fold of NodF

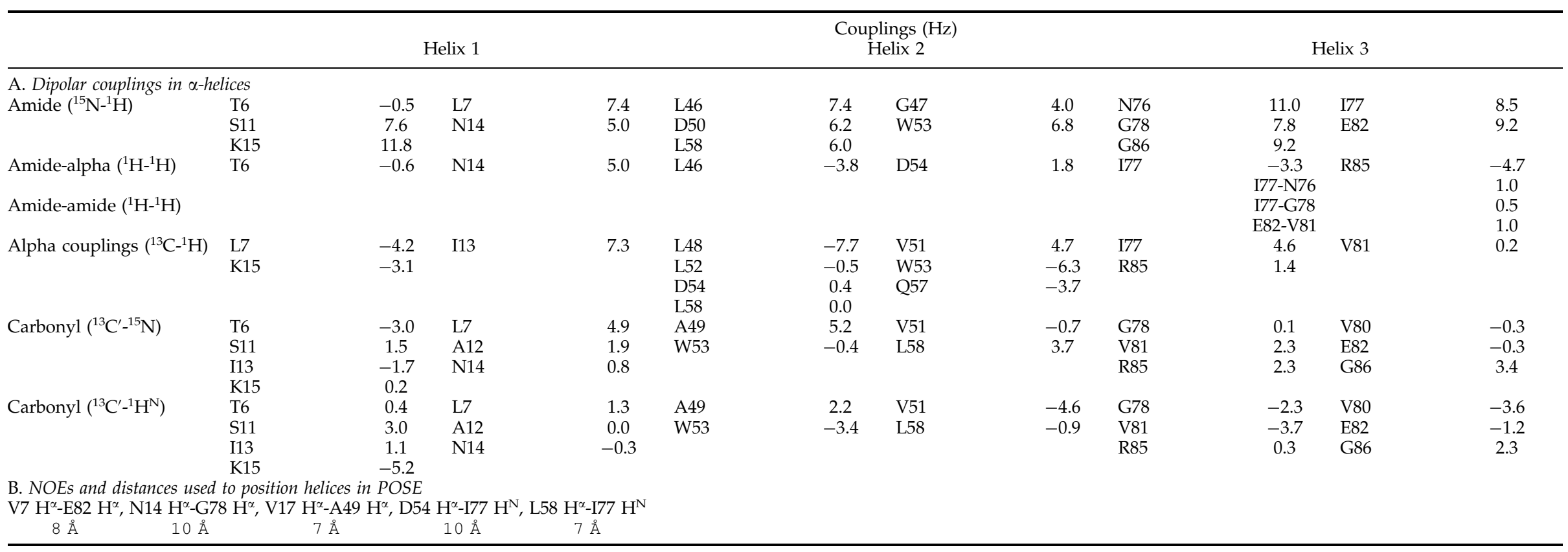


fragment. In the case presented, positions of axes, and in particular the $z$ axis, which corresponds to the direction of highest order, do not appear to be very different when viewed from these standard helices. Therefore, the helices must be nearly parallel, and only small rotations are needed to superimpose order frames for molecular structure assembly.

Before rotating the individual helices into the correct order tensor frame as determined by Orderten-SVD, solutions were filtered for a consistent set of principal order parameters among the three helices. These turned out to be $S_{\mathrm{zz}}$ between -0.001 and -0.0015 and $\eta$ between 0.65 and 0.9 . The correctly aligned helices were then used as input to the program POSE for the determination of a backbone fold. This program was written to translate each rigid structural element through a set of grid points in order to find positions which satisfy a small number of experimental distance constraints that include NOE-derived distances, an approximate maximum extension of intervening loop regions, and the minimum distances for van der Waals contact. All NOE constraints are set to a distance slightly larger than that normally used in more traditional methods $(6-7 \AA$ versus $5 \AA$ maximum), mainly to account for any deviations between true local structures and the idealized polyalanine helices used in our fold determination protocol. For NOEs which involve side-chain protons (in the case of ACP we use some NOEs to the ring of Phe50), the position of the NOE is set to that of the corresponding methyl group in the idealized helices, and an additional 2-3 $\AA$ is added to compensate for the difference in side-chain extension. A complete list of the NOE distance constraints used for ACP is given in Table 1B. Loop distance constraints were set explicitly to a maximum of $2.5 \AA$ times the number of intervening residues. The program repeats the translational search for each of the four possible orientations of each structural element. All solutions which satisfy the data are recorded by the program for analysis.

The final backbone fold for ACP is depicted in Figure 3(a); for comparison, Figure 3(b) shows a previously determined NOE structure of ACP (PDB ID: 1ACP) in approximately the same orientation. ${ }^{36}$ The fold is the average of an ensemble of approximately 900 structures with an RMSD of $2.5 \AA$. A second set of solutions was also produced, but it did not correspond to a closely packed structure and was discarded. In addition to positioning the three helices, we have managed to collect enough dipolar coupling data to orient a type I reverse $\beta$-turn, from residues Thr23 to Ala26, located in the very long loop region between helices 1 and 2 in ACP. We were only able to observe a single NOE, which was insufficient to position this small (four-residue) polypeptide unambiguously with respect to the helices in three-dimensional space; however, one of the four allowed orientations is within approximately
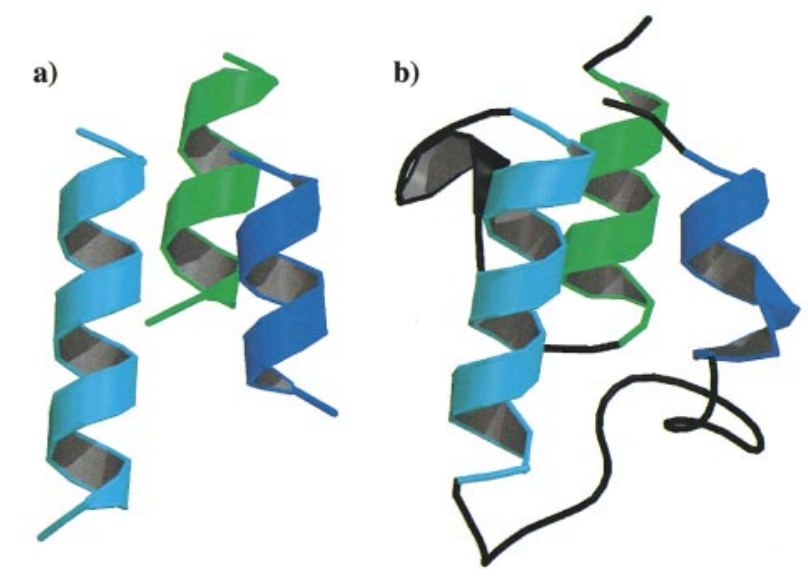

Figure 3. Comparison of ACP structures. (a) Backbone fold as determined from residual dipolar couplings and five long-range NOEs. (b) High-resolution NOE structure. $^{32}$

$15^{\circ}$ of the orientation of this turn in the original structure. $^{36}$

The backbone fold of NodF can be seen in Figure 4 . In this case, each helix was treated as three individual, smaller helices of roughly equal length. These were oriented individually using Orderten-SVD and then reassembled back into three longer helices. Splitting the helices into multiple smaller segments was possible for NodF because of the inclusion of ${ }^{1} \mathrm{D}_{\mathrm{CH}},{ }^{1} \mathrm{D}_{\mathrm{NC}}$, and ${ }^{2} \mathrm{D}_{\mathrm{HC}}$ couplings in addition to the ${ }^{1} \mathrm{D}_{\mathrm{NH}}$ and $\mathrm{D}_{\mathrm{HH}}$ couplings used in ACP. This has an advantage in that it allows for a better fit when the larger structural element deviates from ideality; in fact, splitting the helices was required in this case to get solutions out of Orderten-SVD using reasonable error estimates. The inversion problem did not present any difficulties when recombining the shorter fragments back into full-length helices; in all cases, only one of the four allowed orientations maintains the correct helical twist. This method does, however, lead deviations from regular helix geometry to concentrate at the reattachment sites. Solutions from Orderten-SVD were checked for a consistent set of order parameters, and all three helices had a set of solutions with $S_{\mathrm{zz}}$ ranging from -0.001 to -0.0015 and $\eta$ from 0.5 to 0.65 . The concatenated helices were then positioned in the program POSE, as previously described for $\mathrm{ACP}$, using the NOE distance constraints shown in Table $2 \mathrm{~B}$ and loop maximum distance constraints of $2.5 \AA$ times the number of amino acid residues in the loop. This yielded a single ensemble of 303 allowable structures with an RMSD for the backbone heavy atoms of $2.3 \AA$. A high-resolution structure of NodF using NOEs in addition to dipolar couplings is currently being investigated but is not yet available for comparison. 


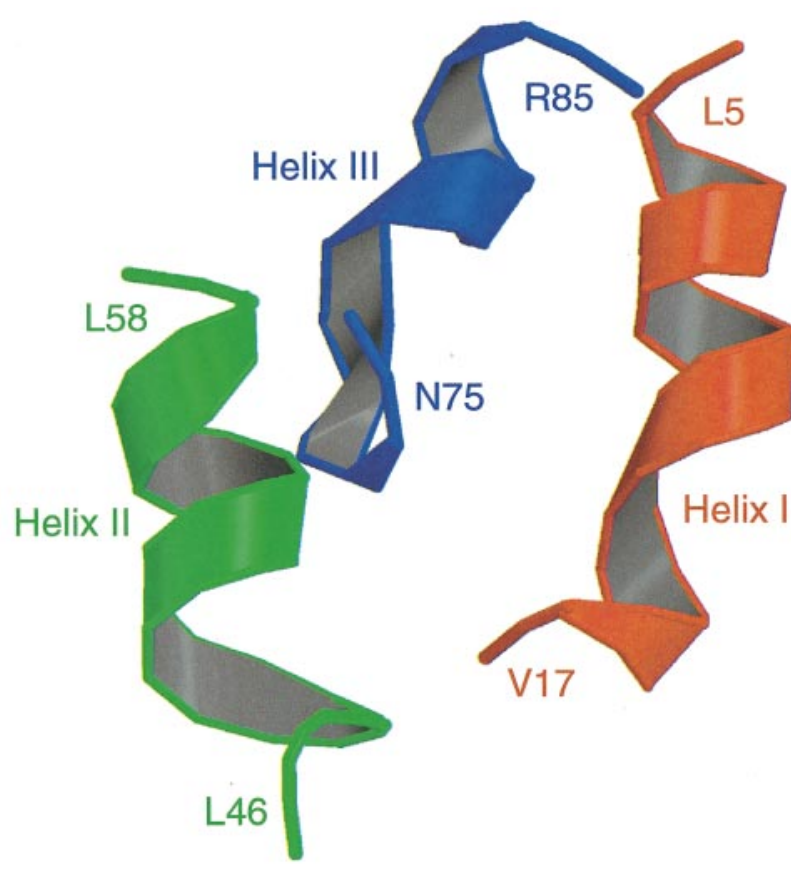

Figure 4. Backbone fold of NodF as determined from residual dipolar couplings and five long-range NOEs.

\section{Discussion}

In traditional, NOE-based structure determination using NMR, the vast majority of time is spent at the stage of data acquisition and analysis, with the goal being the ability to obtain assignments for resonances that are as complete as possible. This often requires identification of poorly resolved resonances for side-chain protons in addition to sequential backbone connectivities. As a result, it is not atypical to spend more than one month collecting and interpreting data before structure calculations can begin.

In contrast, the backbone assignments needed for the determination of a backbone fold can be done relatively quickly. When a doubly ${ }^{13} \mathrm{C},{ }^{15} \mathrm{~N}-$ labeled sample is available, a backbone walk using $\mathrm{HNCACB}$ and $\mathrm{CBCA}(\mathrm{CO}) \mathrm{NH}$ experiments can be performed. ${ }^{39,40}$ Our experiments on a $2.5 \mathrm{mM}$ NodF sample required only two days. When only a singly ${ }^{15} \mathrm{~N}$-labeled sample is available, assignments come from a pair of ${ }^{15} \mathrm{~N}$ filtered experiments, a TOCSY experiment to classify resonances into spin systems and a NOESY experiment to obtain sequential $(i, i+1)$ connectivities, as well as a few long-range connectivities. ${ }^{23,44}$ An HNHAstyle experiment is also run to identify secondary structural motifs from ${ }^{3} \mathrm{~J}_{\mathrm{HNHA}}$ coupling constants. ${ }^{23}$ Although we used previous assignments in our ACP application, the equivalent experiments on NodF required just over four days.

Most experiments for the actual collection of dipolar coupling data are also straightforward to run and relatively efficient in terms of time. ${ }^{45-49}$
We actually acquired a number of redundant data sets in these initial applications. However, we can estimate times corresponding to a minimal set of experiments. For ACP, an oriented phase CTCOSY experiment plus the phase encoded ${ }^{15} \mathrm{~N}-{ }^{1} \mathrm{H}$ HSQC would have required approximately two days. For NodF, phase encoded ${ }^{15} \mathrm{~N}-{ }^{1} \mathrm{H}$ HSQC, CTCOSY, in-phase/anti-phase HSQC, and $\mathrm{C}^{\alpha}-\mathrm{H}^{\alpha}$ HSQCs with four different mixing times would have required only three days. Hence, total data acquisition times can be kept to less than one week with current instrumentation; times should be even shorter using recent advances in NMR hardware.

In order to use our dipolar coupling data to produce a backbone fold, we did have to make a number of assumptions. The first and most important of these is that secondary structural elements conform to an idealized structure. For short polypeptide segments this assumption is good; as shown for NodF, larger elements that deviate from ideality can be broken down into smaller fragments, and then the intact elements can be reassembled. We also assumed that the individual elements are rigid, i.e. that there are no large internal motions. Structure determination by aligning order tensor frames is actually quite tolerant to moderate motions, perhaps even more so than NOE-based methods. In the systems investigated, neither assumption seemed to introduce any severe problems with the final folds.

The accuracy of the backbone folds could be a concern. Figure 3(a) illustrates the average positioning of the three helices of E. coli ACP determined using dipolar couplings and POSE. For comparison, the previously determined NOE structure of ACP is shown in Figure 3(b). The overall geometry is apparently quite similar. In fact, the RMSD between the backbone atoms in the helices of both structures is slightly less than $3 \AA$, a resolution similar to that of a low-resolution crystal structure. This level of error between structures is remarkably good considering that the $\mathrm{C}$ terminus of helix 1 in the original NMR structure (on the right in Figure 3) is quite badly kinked and deviates significantly from our assumption of an ideal helix. Furthermore, helix 3 (the central helix in the figure) is twisted by approximately $40^{\circ}$ about the helical axis. This contributes to the RMSD but does not produce easily visualized departures between the two structures. Hence, a fold description is very well reproduced, while actual positions of backbone atoms may suffer moderate displacements.

We can not make a direct comparison to a structure determined by alternate methods for the NodF protein from R. leguminosarum. However, the structure of NodF can be compared to ACP. NodF yields a bundle of three helices in which helix 1 is anti-parallel to the other two helices, as are the helices in ACP. The similarity is not surprising, as NodF has a reasonably high level of both sequence and functional homology with $\mathrm{ACP}$, especially around the point where the 
phosphopantetheine prosthetic group is attached (Ser36 in ACP, Ser45 in NodF). In each case this site is in a conserved Asp-Ser-Leu motif and the leucine residue begins helix 2. Furthermore, ACP has a hydrophobic cleft between helices 2 and 3 in which an acyl chain can lie. ${ }^{50}$ In both structures a number of hydrophobic residues line the contact area between these helices (F50, I54, A59, V65, A68 and Y71 in ACP; A49, W53, I77, V81, and V84 in NodF), implying that this same motif may exist in NodF.

We can also compare E. coli ACP and NodF to structures of other acyl carrier proteins. One of these is the actinorhodin acyl carrier protein (act ACP) from the polyketide synthase of Streptomyces coelicolor, for which the structure of the apoprotein has been solved using NOE-based methods. ${ }^{51}$ This structure also has three helices (residues 7-16, 4253, and 72-85) arranged in a similar three-helix bundle. Another comparison is with the fatty acid synthase ACP from spinach. The structure for spinach $\mathrm{ACP}$ was derived using homology constraints from E. coli ACP combined with a limited set of NOEs. ${ }^{52}$ The structure again indicates a three-helix bundle with a number of hydrophobic residues that could provide a site for acyl chain stabilization in the area between the second and third helices. Very recently a crystal structure of the holo-ACP synthetase from Bacillus subtilis appeared which contains an ACP molecule in complex. ${ }^{53}$ Here too the general fold of the three major helices seems to be preserved.

In the examples presented, we have dealt only with helical proteins. Application to a wider range of orientable secondary structural elements is of interest. The orienting of a reverse turn in ACP was mentioned in the results, and while there were too few observed distance constraints to correctly position this fragment, it provides some support for application of our method to non-helical structures.

One last issue that remains to be discussed is how useful such a fold can be. Classification of proteins using homology searches has a long history and continues to play an important role today. At low levels of sequence homology, models of backbone structures are the primary result of these methods. Experimental backbone structures can supplement and replace models generated in this way. They can add to, or be searched against in, databases of structural motifs for proteins such as SCOP or CATH. ${ }^{54,}{ }^{55}$ Several tools also exist for comparing features of backbone structures or for extending backbone structures to more complete models; some examples include DALI, WHAT IF, and TOP. ${ }^{56,57,58}$ One recent tool of note employs "fuzzy functional forms" (FFFs) ${ }^{6,}{ }^{59}$ FFFs basically define a conserved geometric placement of residues within a set of related enzymes, typically represented by the positions of backbone atoms, which are not necessarily sequential, but tend to lie in or near the active site. An attempt to identify function is then made by searching for this pattern. The target for the search is a backbone structure, usually produced by sequence homology modeling with an accuracy of 4 to $6 \AA$. Our approach produces more accurate structures without the need for homology. Thus, we are optimistic about the potential utility of experimental backbone folds in structural genomics applications.

\section{Materials and Methods}

\section{Protein purification and sample preparation}

${ }^{15} \mathrm{~N}$-labeled E. coli acyl carrier protein (ACP) was expressed and isolated using a procedure modified from previously reported methods. ${ }^{60-62}$ Briefly, ACP was overexpressed in E. coli BL-21(DE3), and the IPTGinduced cells were harvested and frozen. Thawed and resuspended cells were disrupted by sonication, centrifuged, and most other proteins were removed from the resulting supernatant by the addition of ammonium sulfate to a final concentration of $0.37 \mathrm{~g} / \mathrm{ml}$. The denatured proteins were pelleted, and ACP was precipitated from this supernatant by adjusting the $\mathrm{pH}$ to 3.5 by the dropwise addition of concentrated $\mathrm{HCl}$. ACP was resuspended and treated with $0.2 \mathrm{M}$ hydroxylamine- $\mathrm{HCl}$ at pH 9.0 to strip off any acyl chains. The $\mathrm{pH}$ was lowered to 6.0 and the protein purified over a Q-Sepharose column (Pharmacia, Piscatway, NJ) using a linear gradient from $0.15 \mathrm{M}$ to $1.0 \mathrm{M} \mathrm{NaCl}$; $\mathrm{ACP}$ containing fractions were identified by SDS-PAGE. At this point, E. coli holoacyl carrier protein synthase was expressed and partially purified by batch treatment with DEAE-cellulose (DE-52, Whatman, Clifton, NJ) followed by cation exchange chromatography of the supernatant over an SP-Sepharose column (Pharmacia) eluted with a linear gradient of $\mathrm{NaCl}$ from $0 \mathrm{M}$ to $1 \mathrm{M}{ }^{63,64}$ The resulting crude synthase was used to convert mixed ACP entirely to the holo form using coenzyme A (Sigma, St. Louis, MO) as the phosphopantetheine source. ${ }^{63,64}$ Following incubation of both proteins with coenzyme A, conversion to holo-ACP was verified by SDS-PAGE. Conversion to holo-protein was judged greater than $95 \%$, and product was recovered from the reaction mixture using a Q-Sepharose column eluted with $0.5 \mathrm{M} \mathrm{NaCl}$. Finally, the holo-ACP containing fractions, as determined by SDS-PAGE, were purified by gel filtration HPLC using a TSK-750 column (Bio-Rad, Hercules, CA).

${ }^{13} \mathrm{C}_{1}^{15} \mathrm{~N}$ NodF was prepared by Dr Otto Geiger as outlined. ${ }^{37}$ In short, IPTG-induced cells were harvested, frozen, resuspended, and lysed by passage through a French pressure cell. Ethanol was added dropwise while stirring at $4{ }^{\circ} \mathrm{C}$ to a final concentration of $50 \%(\mathrm{v} / \mathrm{v})$ and the solution incubated for an additional hour. Precipitated proteins were removed by centrifugation and the supernatant dialyzed to remove ethanol. The NodF from the dialysate was further purified using a DEAE-cellulose column (DE-52) eluted with a linear gradient from $0.1 \mathrm{M}$ to $1.0 \mathrm{M} \mathrm{NaCl}$. NodF containing fractions were determined using SDS-PAGE.

Pf1 bacteriophage, used as an orienting medium for NMR experiments, was prepared as outlined. ${ }^{41,65}$ Pseudomonas aeruginosa was grown on LB to an $A_{600}$ of $\sim 1.0$, infected with Pf1, and grown for an additional 16 hours. Host cells were removed by centrifugation and the Pf1 precipitated by adding $60 \mathrm{~g} / 1$ of $\mathrm{NaCl}$ and $20 \mathrm{~g} / 1$ of PEG-8000 while stirring on ice. The phage was collected by centifugation. Phage was then purified by ultracentrifugation using a $\mathrm{KBr}$ density gradient; a step gradient 
from $20 \%$ to $50 \% \mathrm{KBr}(\mathrm{w} / \mathrm{v})$ was used, with the phage loaded in the $20 \%$ layer. After centrifugation, the visible faint blue band of phage near the middle of the gradient was carefully extracted by syringe and then extensively dialyzed against TE buffer $(10 \mathrm{mM}$ Tris, $1 \mathrm{mM}$ EDTA ( $\mathrm{pH} 7.0)$ ) to remove $\mathrm{KBr}$. Finally, Pf1 was pelleted by ultracentrifugation and the pellet stored at $4{ }^{\circ} \mathrm{C}$ until needed.

Final NMR samples of ACP and NodF were obtained by concentrating and exchanging buffer using Centricon3 devices (Amicon, Beverly, MA). ${ }^{15} \mathrm{~N}$ holo-ACP samples were $25 \mathrm{mM}$ bis-Tris in $10 \%{ }^{2} \mathrm{H}_{2} \mathrm{O}, 90 \% \mathrm{H}_{2} \mathrm{O}(\mathrm{pH} 6.0)$, containing $10 \mathrm{mM} \mathrm{CaCl}$ for protein stability (protein concentrations were $2 \mathrm{mM}$ isotropic and $1 \mathrm{mM}$ aligned). ${ }^{13} \mathrm{C},{ }^{15} \mathrm{~N}$ NodF samples were $200 \mathrm{mM}$ potassium phosphate in $10 \%{ }^{2} \mathrm{H}_{2} \mathrm{O}, 90 \% \mathrm{H}_{2} \mathrm{O}(\mathrm{pH}$ 6.1) with no other salts present (protein concentrations were $2.5 \mathrm{mM}$ isotropic and $1 \mathrm{mM}$ aligned). Trace amounts of sodium azide were added to all samples as a preservative. Alignment of samples for the observation of dipolar couplings was achieved using Pf1 filamentous bacteriophage. ${ }^{41}$ Samples with the correct volume and buffer conditions were lyophilized and then rehydrated with enough aqueous phage to give a ${ }^{2} \mathrm{H}_{2} \mathrm{O}$ quadrupolar splitting of $12-18 \mathrm{~Hz}$ (approximately $20 \mathrm{mg} / \mathrm{ml}$ phage).

\section{NMR spectroscopy}

All NMR spectra were acquired on Varian Inova spectrometers operating at 500, 600, or $800 \mathrm{MHz}$ and equipped with $5 \mathrm{~mm}$ triple resonance gradient probes. Experiments run on each sample for both assignment and the observation of couplings, including experimental conditions, are listed in Table 3; examples of some of the spectra can be found in Supplementary Material. ACP side-chain assignments and ${ }^{3} J_{\mathrm{HNHA}}$ scalar couplings were previously reported by Prestegard and co-workers using different, COSY based methods and are not reported again here. ${ }^{31,32,36}$ Peak positions were determined and intensities or volumes were measured using the routines built into the software package Felix (MSI, San Diego, CA).

\section{Measurement of scalar and residual dipolar couplings}

A large number of different couplings were measured for the determination of a backbone fold; we briefly present here the methods by which couplings can be extracted from the various NMR spectra used. ${ }^{1} J_{\mathrm{NH}}$ and ${ }^{1} \mathrm{D}_{\mathrm{NH}}$ couplings were measured in two ways. A coupling-enhanced HSQC experiment was used, ${ }^{66}$ in which the couplings can simply be measured by dividing the difference of two peak centers by two. For our purposes, measuring the couplings from the positions of peak maxima, as interpolated by Felix, has a high enough level of precision (on the order of $\pm 1.0 \mathrm{~Hz}$ ). A phase-encoded HSQC experiment was also used. ${ }^{45}$ In this case, two different spectra are acquired in an interleaved fashion with the coupling modulated by either a sine or cosine function during the evolution time, $T$. The coupling can be extracted using the following formula:

$$
\Delta J=\frac{\arctan \left(\frac{V_{\text {sin }}}{V_{\text {cos }}}\right)}{\pi T}
$$

where $\Delta J$ is the difference between the true scalar (or dipolar) coupling and an experimental tune frequency (typically $93-95 \mathrm{~Hz}$ for ${ }^{15} \mathrm{~N}-{ }^{1} \mathrm{H}$ couplings) and $V_{\text {sin }}$ and $V_{\text {cos }}$ are the volumes of the peaks in the sine and cosine experiments, respectively. Errors on the measurement of couplings using this method were typically less than $\pm 0.5 \mathrm{~Hz}$.

${ }^{3} J_{\text {HNHA }}$ couplings were measured using either an HNHA experiment ${ }^{35}$ or CT-COSY ${ }^{46}$ experiments. For the HNHA experiment, the couplings can be extracted from the intensities of the auto- and cross-peaks using the following relation ${ }^{35}$ :

$$
\frac{I_{\text {cross }}}{I_{\text {auto }}}=-\tan ^{2}\left(\pi J_{\mathrm{HH}} t_{\text {mix }}\right)
$$

in which $I_{\text {cross }}$ and $I_{\text {auto }}$ are the cross-peak and auto-peak intensities, $J_{\mathrm{HH}}$ is the HNHA coupling of interest, and $t_{\text {mix }}$ the mixing time of the experiment.

The following equation can be used to extract ${ }^{3} J_{\mathrm{HNHA}}$ as well as ${ }^{3} \mathrm{D}_{\mathrm{HNHA}}$ and any other observable ${ }^{1} \mathrm{H}-{ }^{1} \mathrm{H}$ dipolar couplings, from CT-COSY experiments ${ }^{46,67}$ :

$$
J=\frac{\arctan \left(\frac{I_{\text {cross }}}{I_{\text {auto }}}\right)}{\pi T}
$$

Here $J$ is once again the scalar or dipolar coupling being measured, $I_{\text {cross }}$ and $I_{\text {auto }}$ are the cross-peak and autopeak intensities, and $T$ is constant time during which the coupling evolves. Because intensities are difficult to measure as volumes for the antiphase cross-peaks, two values of $T$ were used, which allowed a scaling factor to be introduced in equation (6) so that peak height ratios could accurately represent intensity ratios.

In the case of doubly ${ }^{13} \mathrm{C}_{1}^{15} \mathrm{~N}$ labeled NodF, we measured an additional set of couplings involving ${ }^{13} \mathrm{C}$. $\mathrm{C}^{\alpha} \mathrm{H}^{\alpha}$ couplings are measured using a J-modulated constant-time HSQC experiment. ${ }^{47}$ In this experiment, intensities of the observed peaks are proportional to a cosine function:

$$
I \propto \cos \left[2 \pi^{1} J_{\mathrm{CH}}(T-\Delta)\right]
$$

in which $T$ is the constant time required to refocus undesired carbon-carbon couplings and $\Delta$ is a slight change to this delay which modulates the intensities of the observed peaks. In actuality the coupling is extracted by optimizing the fit of the cosine function to a number of intensities from a set of experiments run with different values for $\Delta$. Finally, an in-phase, anti-phase experiment was run to obtain values for ${ }^{1} \mathrm{D}_{\mathrm{NC}^{\prime}}$ and ${ }^{2} \mathrm{D}_{\mathrm{HC}^{\prime}}{ }^{48}$ These sets of couplings are extracted simply by measuring the differences between the centers of two peaks in a multiplet; the one bond ${ }^{15} \mathrm{~N}-{ }^{13} \mathrm{C}^{\prime}$ coupling is measured in the indirect dimension while the displacement in the direct

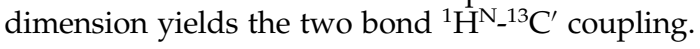

\section{Fold determination}

For the determination of a protein backbone fold, the molecular orientation frame (also called the principal alignment frame) for each rigid fragment was determined by singular value decomposition using the program Orderten-SVD. ${ }^{20}$ In the case of ACP, each helix was treated as a single rigid entity; in determining the fold of NodF, each helix was split into three pieces with roughly equivalent numbers of couplings for each smaller fragment, followed by reassembly of the fragments back into full-length helices. The starting coordinates for 
Table 3. Experiments run on ACP and NodF for backbone assignments and the measurement of residual dipolar couplings

\begin{tabular}{|c|c|c|c|c|c|c|c|c|}
\hline Experiment & Reference & & $\begin{array}{l}\text { Time } \\
(\mathrm{h})\end{array}$ & $\begin{array}{l}t_{\operatorname{mix}} \\
(\mathrm{ms})\end{array}$ & $\begin{array}{l}\text { Field } \\
(\mathrm{MHz})\end{array}$ & $\begin{array}{l}\text { No. of } \\
\text { points }\end{array}$ & $\begin{array}{l}\text { Sweep width } \\
(\mathrm{Hz})\end{array}$ & $\begin{array}{l}\text { Final size } \\
\text { (pts) }\end{array}$ \\
\hline \multicolumn{9}{|l|}{ A. ${ }^{15} \mathrm{~N}$-ACP experiments } \\
\hline NOESY- ${ }^{15} \mathrm{~N}-\mathrm{HSQC}$ & 34 & Isotropic & 38 & 150 & 600 & $1024 \times 158 \times 18$ & $6000 \times 6000 \times 2000$ & $512 \times 512 \times 64$ \\
\hline Intensity modulated & 45 & Isotropic & 1.5 & 43 & 600 & $512 \times 128$ & $6000 \times 2200$ & $1024 \times 512$ \\
\hline${ }^{15} \mathrm{~N}-\mathrm{HSQC}$ & & Aligned & 3.0 & 43 & 600 & $512 \times 128$ & $6000 \times 2200$ & $1024 \times 512$ \\
\hline \multirow[t]{4}{*}{${ }^{15} \mathrm{~N}-\mathrm{HMQC}-\mathrm{CT}-\mathrm{COSY}$} & 46 & Isotropic & 20 & 25 & 800 & $1024 \times 48 \times 18$ & $10,650 \times 5600 \times 3200$ & $1024 \times 128 \times 64$ \\
\hline & & & 20 & 35 & 800 & $1024 \times 48 \times 18$ & $10,650 \times 5600 \times 3200$ & $1024 \times 128 \times 64$ \\
\hline & & Aligned & 20 & 25 & 800 & $1024 \times 48 \times 18$ & $10,650 \times 5600 \times 3200$ & $1024 \times 128 \times 64$ \\
\hline & & & 20 & 35 & 800 & $1024 \times 48 \times 18$ & $10,650 \times 5600 \times 3200$ & $1024 \times 128 \times 64$ \\
\hline \multicolumn{9}{|c|}{ B. ${ }^{13} \mathrm{C}^{15} \mathrm{~N}-\mathrm{NodF}$ experiments } \\
\hline NOESY- ${ }^{-15} \mathrm{~N}-\mathrm{HSQC}$ & 34 & Isotropic & 48 & 150 & 800 & $1024 \times 148 \times 24$ & $9600 \times 9600 \times 2920$ & $1024 \times 512 \times 64$ \\
\hline TOCSY- ${ }^{15} \mathrm{~N}-\mathrm{HSQ} \mathrm{C}$ & 69 & Isotropic & 32 & 70 & 500 & $512 \times 128 \times 30$ & $6000 \times 1330 \times 3000$ & $512 \times 256 \times 64$ \\
\hline HNHA & 35 & Isotropic & 20 & 26 & 800 & $512 \times 32 \times 24$ & $9200 \times 9200 \times 3200$ & $512 \times 128 \times 64$ \\
\hline $\mathrm{CBCA}(\mathrm{CO}) \mathrm{NH}$ & 70,71 & Isotropic & 16 & & 600 & $512 \times 52 \times 24$ & $7200 \times 9050 \times 2400$ & $512 \times 128 \times 64$ \\
\hline HNCACB & 71,72 & Isotropic & 16 & & 600 & $512 \times 52 \times 24$ & $7200 \times 9050 \times 2400$ & $512 \times 128 \times 64$ \\
\hline $\mathrm{HNCO}$ & 71,73 & Isotropic & 12 & & 600 & $512 \times 48 \times 24$ & $7200 \times 2420 \times 2400$ & $512 \times 128 \times 64$ \\
\hline \multirow{2}{*}{$\begin{array}{l}\text { Phase encoded } \\
{ }^{15} \mathrm{~N}-\mathrm{HSQC}\end{array}$} & 45 & Isotropic & 1.5 & 43 & 600 & $512 \times 128$ & $6000 \times 2200$ & $1024 \times 512$ \\
\hline & & Aligned & 2.0 & 22 & 800 & $1024 \times 96$ & $9620 \times 2800$ & $1024 \times 512$ \\
\hline \multirow{2}{*}{$\begin{array}{l}\text { Coupling enhanced } \\
{ }^{15} \mathrm{~N}-H S Q C\end{array}$} & 66 & Isotropic & 3.0 & & 600 & $512 \times 256$ & $7200 \times 2400$ & $1024 \times 512$ \\
\hline & & Aligned & 1.5 & & 600 & $512 \times 128$ & $7200 \times 2400$ & $1024 \times 512$ \\
\hline \multirow{2}{*}{${ }^{15} \mathrm{~N}-\mathrm{HMQC}-\mathrm{CT}-\mathrm{COSY}$} & 46 & Aligned & 18 & 25 & 800 & $512 \times 48 \times 32$ & $9620 \times 5600 \times 2800$ & $1024 \times 128 \times 64$ \\
\hline & & & 18 & 40 & 800 & $512 \times 48 \times 32$ & $9620 \times 5600 \times 2800$ & $1024 \times 128 \times 64$ \\
\hline Intensity modulated & 47 & Isotropic & 6 ea. & see legend & 800 & $512 \times 256$ & $8000 \times 20000$ & $2048 \times 1024$ \\
\hline${ }^{13} \mathrm{C}-\mathrm{HSQCC}$ & & Aligned & 8 ea. & see legend & 500 & $1024 \times 80$ & $6500 \times 12000$ & $2048 \times 1024$ \\
\hline IPAP ${ }^{15} \mathrm{~N}-{ }^{13} \mathrm{C}^{\prime}$ & 48 & Isotropic & 6 & & 800 & $1024 \times 512$ & $9600 \times 2920$ & $1024 \times 1024$ \\
\hline${ }^{1} \mathrm{H}^{\mathrm{N}}-13 \mathrm{C}^{\prime}$ & & Aligned & 12 & & 800 & $1024 \times 480$ & $9600 \times 2920$ & $1024 \times 1024$ \\
\hline
\end{tabular}

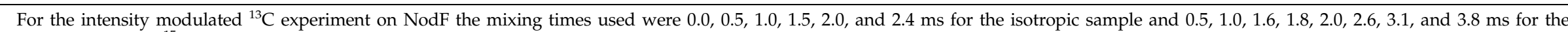
aligned sample. For ${ }^{15} \mathrm{~N}$-edited experiments, the final matrix size indicated is that after the upfield half of the data was discarded in the direct dimension. 
each fragment came from an idealized polyalanine starting structure generated using standard values for the backbone $\phi, \psi$, and $\omega$ torsion angles. ${ }^{42}$ The molecular fragments were rotated such that the principle axis systems of each of their orientation frames were coincident.

The oriented fragments were translated with respect to each other in three-dimensional space using a new program, POSE (positioning of structural elements) and a minimal set of NOEs (two to three distance constraints per rigid element, see Tables 1 and 2). The program will be made available on our website: http://tesla.ccrc.uga.edu. A general outline of how the program works is given in the flowchart in Figure 5. Briefly, any two of the correctly oriented structural elements are provided as input to the program along with a list of NOEs and other distance constraints (for example, a short three or fourresidue loop would force two elements which pack closely together into an anti-parallel orientation). One of the atoms involved in an NOE is chosen as a starting point, and a spherical grid of possible solutions to that NOE is generated about it. The second element is then translated so that the atom connected by the NOE superimposes on the first point of the grid. A check is made to assure that any remaining NOEs and other distance constraints are satisfied, and then an additional check is made for van der Waals collisions between every possible pair of atoms. If there are any violations of distance constraints or close contacts, the solution is rejected; if all conditions are satisfied, the solution is allowed and written to a file. The program runs until all points in the grid are tested, appending each possible solution to the output file. If the inversion problem that arises from our inability to distinguish the positive and negative directions for each axis of the alignment frame has not previously been solved using a second aligning medium, ${ }^{43}$ the process is repeated for all four allowed inversions of the starting structure of the second element. $\mathrm{Up}$ to this point, the entire process required very little computational time (less than two minutes using an SGI Indy with a $150 \mathrm{MHz}$ R4400 processor). In order to position the third helix, the first two helices were combined into a new "first" element, the third helix was added as the "second" element, and these were used along with an appropriate set of distance constraints as inputs to POSE. In order to assure that we did not throw away any valid solutions, we repeated the process using every allowed solution for the first pair of elements. Additional elements can be added by repeating the entire process in an iterative fashion.

Coordinates for backbone atoms of secondary structure elements in NodF are being deposited in the RCSB Protein Data Bank ${ }^{68}$ with accession number 1FH1.

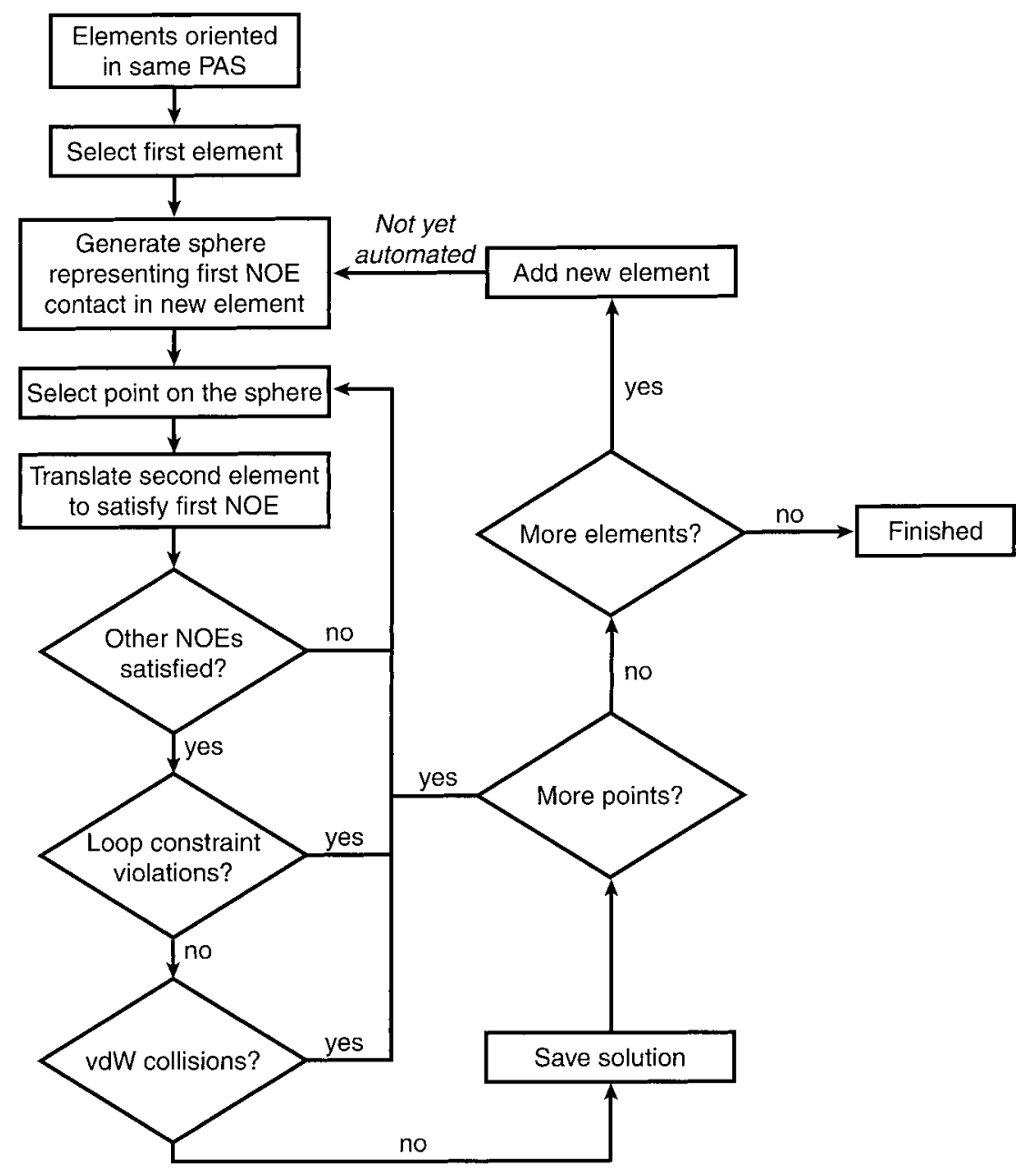

Figure 5. Flow chart illustrating the operation of the program POSE. 


\section{Acknowledgments}

We would like to thank Dr Otto Geiger for preparing the sample of NodF, and thank Bryan Woosley and Dr Edward Zartler for providing the Pf1 bacteriophage. Funding was provided by a grant from the National Science Foundation (MCB 9726341).

\section{References}

1. Sali, A. \& Kuriyan, J. (1999). Challenges at the frontiers of structural biology. Trends Biol. Sci. 24, M20-M24.

2. Zarembinski, T. I., Hung, L. W., Mueller-Dieckman, H. J., Kim, K. K., Yokota, H., Kim, R. \& Kim, S. H. (1998). Structure-based assignment of the biochemical function of a hypothetical protein: a test case of structural genomics. Proc. Natl Acad. Sci. USA, 95, 15189-15193.

3. Sali, A. (1998). 100,000 protein structures for the biologist. Nature Struct. Biol. 5, 1029-1032.

4. Montelione, G. T. \& Anderson, S. (1999). Structural genomics: keystone for a Human Proteome Project. Nature Struct. Biol. 6, 11-12.

5. Skolnick, J., Fetrow, J. S. \& Kolinski, A. (2000). Structural genomics and its importance for gene function analysis. Nature Biotechnol. 18, 283-287.

6. Skolnick, J. \& Fetrow, J. S. (2000). From genes to protein structure and function: novel applications of computational approaches in the genomic era. Trends Biotechnol. 18, 34-39.

7. Holm, L. \& Sander, C. (1996). Mapping the protein universe. Science, 273, 595-602.

8. Holm, L. \& Sander, C. (1994). Searching protein structure databases has come of age. Proteins: Struct. Funct. Genet. 19, 165-173.

9. Gibrat, J. F., Madej, T. \& Bryant, S. H. (1996). Surprising similarities in structure comparison. Curr. Opin. Struct. Biol. 6, 377-385.

10. Garrett, D. S., Powers, R., Gronenborn, A. M. \& Clore, G. M. (1991). A common sense approach to peak picking in two, three, and four-dimensional spectra using automatic computer analysis of contour diagrams. J. Magn. Reson. 95, 214-220.

11. Koradi, R., Billeter, M., Engeli, M., Güntert, P. \& Wüthrich, K. (1998). Automated peak picking and peak integration in macromolecular NMR spectra using AUTOPSY. J. Magn. Reson. 135, 288-297.

12. Zimmerman, D. E., Kulikowski, C. A., Huang, Y., Feng, W., Tashiro, M., Shimotakahara, S., Chien, C. Y., Powers, R. \& Montelione, G. T. (1997). Automated analysis of protein NMR assignments using methods from artificial intelligence. J. Mol. Biol. 269, 592-610.

13. Gronwald, W., Willard, L., Jellard, T., Boyko, R. F., Krishna, R., Wishart, D. S., Sönnichsen, F. D. \& Sykes, B. D. (1998). CAMRA: chemical shift based computer aided protein NMR assignments. J. Biomol. NMR, 12, 395-405.

14. Bartels, C., Güntert, P., Billeter, M. \& Wüthrich, K. (1997). GARANT - A general algorithm for resonance assignment of multidimensional nuclear magnetic resonance spectra. J. Comput. Chem. 18, 139149.

15. Nilges, M., Macias, M. J., O’Donoghue, S. I. \& Oschkinat, H. (1997). Automated NOESY interpretataion with ambiguous distance restraints: the refined NMR solution structures of the pleckstring hom- ology domain from $\beta$-spectrin. J. Mol. Biol. 269, 408422.

16. Nilges, M. \& O’Donoghue, S. I. (1998). Ambiguous NOEs and automated NOE assignment. Prog. Nucl. Magn. Reson. Spect. 32, 107-139.

17. Tjandra, N. \& Bax, A. (1997). Direct measurement of distances and angles in biomolecules by NMR in a dilute liquid crystalline medium. Science, 278, 11111114.

18. Prestegard, J. H., Al-Hashimi, H. M. \& Tolman, J. R. (2000). NMR structures of biomolecules using field oriented media and residual dipolar couplings. Quart. Rev. Biophys. In the press.

19. Fischer, M. W. F., Losonczi, J. A., Weaver, J. L. \& Prestegard, J. H. (1998). Domain orientation and dynamics in multidomain proteins from residual dipolar couplings. Biochemistry, 38, 9013-9022.

20. Losonczi, J. A., Andrec, M., Fischer, M. W. F. \& Prestegard, J. H. (1999). Order matrix analysis of residual dipolar couplings using singular value decomposition. J. Magn. Reson. 138, 334-342.

21. Clore, G. M. \& Gronenborn, A. M. (1998). New methods of structure refinement for macromolecular structure determination by NMR. Proc. Natl Acad. Sci. USA, 95, 5891-5898.

22. Delaglio, F., Kontaxis, G. \& Bax, A. (2000). Protein structure determination using molecular fragment replacement and NMR dipolar couplings. J. Am. Chem. Soc. 122, 2142-2143.

23. Wüthrich, K. (1986). NMR of Proteins and Nucleic Acids, John Wiley and Sons, New York.

24. Wishart, D. S., Sykes, B. D. \& Richards, F. M. (1992). The chemical shift index: a fast and simple method for the assignment of protein secondary structure through NMR spectroscopy. Biochemistry, 31, 16471651.

25. Saupe, A. (1968). Recent results in the field of liquid crystals. Angewandte Chemie Int. (Edit. English), 7, 97112.

26. Magnuson, K., Jackowski, S., Rock, C. O. \& Cronan, J. E., Jr (1993). Regulation of fatty acid biosynthesis in Eschericia coli. Microbiol. Rev. 57, 522-542.

27. Vanden, Boom T. \& Cronan, J. E., Jr (1989). Genetics and regulation of bacterial lipid metabolism. Annu. Rev. Microbiol. 43, 317-343.

28. Spaink, H. P., Sheeley, D. M., van Brussel, A. A. N., Glushka, J., York, W. S., Tak, T., Geiger, O., Kennedy, E. P., Reinhold, V. N. \& Lugtenberg, B. J. J. (1991). A novel highly unsaturated fatty acid moiety of lipo-oligosaccharide signals determines host specificity of Rhizobium. Nature, 354, 125-130.

29. Geiger, O., Spaink, H. P. \& Kennedy, E. P. (1991). Isolation of the Rhizobium leguminosarum NodF nodulation protein: NodF carries a 4'-phosphopantetheine prosthetic group. J. Bacteriol. 173, 2872-2878.

30. Ritsema, T., Gehring, A. M., Stuitje, A. R., van der Drift, K. M. G. M., Dandal, I., Lambalot, R. H., Walsh, C. T., Thomas-Oates, J. E., Lugtenberg, B. J. J. \& Spaink, H. P. (1998). Functional analysis of an interspecies chimera of acyl carrier proteins indicates a specialized domain for protein recognition. Mol. Gen. Genet. 257, 641-648.

31. Holak, T. A. \& Prestegard, J. H. (1986). Secondary structure of acyl carrier protein as derived from two-dimensional ${ }^{1} \mathrm{H}$ NMR spectroscopy. Biochemistry, 25, 5766-5774.

32. Kim, Y. \& Prestegard, J. H. (1989). A dynamic model for the structure of acyl carrier protein in solution. Biochemistry, 28, 8792-8797. 
33. Andrec, M. R., Hill, R. B. \& Prestegard, J. H. (1995). Amide exchange rates in Eschericia coli acyl carrier protein: correlation with protein structure and dynamics. Protein Sci. 4, 983-993.

34. Jahnke, W., Baur, M., Gemmecker, G. \& Kessler, H. (1995). Improved accuracy of NMR structures by a modified NOESY-HSQC experiment. J. Magn. Reson. 106, 86-88.

35. Vuister, G. W. \& Bax, A. (1993). Quantitative J correlation: a new approach for measuring homonuclear three-bond $J\left(\mathrm{H}^{\mathrm{N}} \mathrm{H}^{\alpha}\right)$ coupling constants in ${ }^{15} \mathrm{~N}$ enriched proteins. J. Am. Chem. Soc. 115, 7772-7777.

36. Kim, Y. \& Prestegard, J. H. (1990). Refinement of the NMR structures for acyl carrier protein with scalar coupling data. Proteins: Struct. Funct. Genet. 8, 377385.

37. Ghose, R., Geiger, O. \& Prestegard, J. H. (1996). NMR investigations of the structural properties of the nodulation protein, NodF, from Rhizobium leguminosarum and its homology with Escherichia coli acyl carrier protein. FEBS Letters, 388, 66-72.

38. Huang, K., Andrec, M., Heald, S., Blake, P. \& Prestegard, J. H. (1997). Performance of a neuralnetwork-based determination of amino acid class and secondary structure from ${ }^{1} \mathrm{H}^{15} \mathrm{~N}$ NMR data. J. Biomol. NMR, 10, 45-52.

39. Cavanagh, J., Fairbrother, W. J., Palmer, A. G. I. \& Skelton, N. J. (1996). Protein NMR Spectroscopy: Principles and Practice, Academic Press, San Diego.

40. Sattler, M., Schleucher, J. \& Griesinger, C. (1999). Heteronuclear multidimensional NMR experiments for the structure determination of proteins in solution employing pulsed field gradients. Prog. Nucl. Magn. Reson. Spectr. 34, 93-158.

41. Hansen, M. R., Mueller, L. \& Pardi, A. (1998). Tunable alignment of macromolecules by filamentous phage yields dipolar coupling interactions. Nature Struct. Biol. 5, 1065-1074.

42. Creighton, T. E. (1993). Proteins: Structures and Molecular Properties, 2nd edit., W.H. Freeman and Co., New York.

43. Al-Hashimi, H. M., Valafar, H., Terrell, M., Zartler, E. R., Eidsness, M. K. \& Prestegard, J. H. (2000). Variation of molecular alignment as a means of resolving orientational ambiguities in protein structures from dipolar couplings. J. Magn. Reson. 143, 402-406.

44. Clore, G. M. \& Gronenborn, A. M. (1991). Applications of three and four-dimensional heteronuclear NMR spectroscopy to protein structure determination. Prog. Nucl. Magn. Reson. Spectr. 23, 43-92.

45. Tolman, J. R. \& Prestegard, J. H. (1996). A quantitative $J$-correlation experiment for the accurate measurement of one bond amide ${ }^{15} \mathrm{~N}-{ }^{1} \mathrm{H}$ couplings in proteins. J. Magn. Reson. 112 B, 245-252.

46. Tian, F., Fowler, C. A., Zartler, E. R., Jenney, F. A. J., Adams, M. W. \& Prestegard, J. H. (2000). Direct measurement of ${ }^{1} \mathrm{H}-{ }^{1} \mathrm{H}$ dipolar couplings in proteins: a complement to traditional NOE measurements. J. Biomol. NMR, 18, 23-31.

47. Tjandra, N. \& Bax, A. (1997). Measurement of dipolar contributions to ${ }^{1} J_{\mathrm{CH}}$ splittings from magnetic-field dependence of $J$ modulation in twodimensional NMR spectra. J. Magn. Reson. 124, 512515.

48. Wang, Y., Marquardt, J. L., Wingfield, P., Stahl, S. J., Lee-Huang, S., Torchia, D. \& Bax, A. (1998). Simultaneous measurement of ${ }^{1} \mathrm{H}_{-}-{ }^{15} \mathrm{~N},{ }^{1} \mathrm{H}-{ }^{13} \mathrm{C}^{\prime}$, and ${ }^{15} \mathrm{~N}-{ }^{13} \mathrm{C}^{\prime}$ dipolar couplings in a perdeuterated
$30 \mathrm{kDa}$ protein dissolved in a dilute liquid crystalline phase. J. Am. Chem. Soc. 120, 7385-7386.

49. Ottigen, M., Delaglio, F. \& Bax, A. (1998). Measurement of $\mathrm{J}$ and dipolar couplings from simplified two-dimensional NMR spectra. J. Mang. Reson. 131, 373-378.

50. Jones, P. J., Holak, T. A. \& Prestegard, J. H. (1987). Structural comparison of acyl carrier protein in acylated and sulfhydryl forms by two-dimensional ${ }^{1} \mathrm{H}$ NMR spectroscopy. Biochemistry, 26, 3493-3500.

51. Crump, M. P., Crosby, J., Dempsey, C. E., Parkinson, J. A., Murray, M., Hopwood, D. A. \& Simpson, T. J. (1997). Solution structure of the actinorhodin polyketide synthase acyl carrier protein from Streptomyces coelicolor A3(2). Biochemistry, 36, 6000-6008.

52. Oswood, M. C., Kim, Y., Ohlrogge, J. B. \& Prestegard, J. H. (1997). Structural homology of spinach acyl carrier protein and Escherichia coli acyl carrier protein based on NMR data. Proteins: Struct. Funct. Genet. 27, 131-143.

53. Parris, K. D., Lin, L., Tam, A., Mathew, R., Hixon, J., Stahl, M., Fritz, C. C., Seehra, J. \& Somers, W. S. (2000). Crystal structures of substrate binding to Bacillus subtilis holo-(acyl carrier protein) synthase reveal a novel trimeric arrangement of molecules resulting in three active sites. Structure, 8, 883-895.

54. Lo Conte, L., Ailey, B., Hubbard, T. J. P., Brenner, S. E., Murzin, A. G. \& Chothia, C. (2000). SCOP: a structural classification of proteins database. Nucl. Acids Res. 28, 257-259.

55. Pearl, F. M. G., Lee, D., Bray, J. E., Sillitoe, I., Todd, A. E., Harrison, A. P., Thornton, J. M. \& Orengo, C. A. (2000). Assigning genomic sequences to CATH. Nucl. Acids Res. 28, 277-282.

56. Holm, L. \& Sander, C. (1993). Protein structure comparison by alignment of distance matrices. J. Mol. Biol. 233, 123-138.

57. Vriend, G. \& Sander, C. (1991). Detection of common three-dimensional substructures in proteins. Proteins: Struct. Funct. Genet. 11, 52-58.

58. Lu, W. (2000). TOP: a new method for protein structure comparisons and similarity searches. J. Appl. Crystallog. 33, 176-183.

59. Fetrow, J. S. \& Skolnick, J. (1998). Method for prediction of protein function from sequence using the sequence-to-structure-to-function paradigm with application to glutaredoxins/thioredoxins and $\mathrm{T}_{1}$ ribonucleases. J. Mol. Biol. 281, 949-968.

60. Rock, C. O. \& Cronan, J. E., Jr (1980). Improved purification of acyl carrier protein. Anal. Biochem. 102, 362-364.

61. Morris, S. A., Revill, W. P., Staunton, J. \& Leadlay, P. F. (1993). Purification and separation of holo and apo forms of Saccharopolyspora erythraea acyl-carrier protein released from recombinant Eschericia coli by freezing and thawing. Biochem. J. 294, 521-527.

62. Hill, R. B., MacKenzie, K. R., Flanagan, J. M., Cronan, J. E., Jr \& Prestegard, J. H. (1995). Overexpression, purification, and characterization of Eschericia coli acyl carrier protein and two mutant proteins. Protein Express. Purif. 6, 394-400.

63. Lambalot, R. H. \& Walsh, C. T. (1995). Cloning, overproduction, and characterization of the Eschericia coli holo-acyl carrier protein synthase. J. Biol. Chem. 270, 24658-24661.

64. Lambalot, R. H. \& Walsh, C. T. (1997). Holo-[acylcarrier-protein] synthase of Eschericia coli. Methods Enzymol. 279, 254-262. 
65. Hansen, M. R., Hanson, P. \& Pardi, A. (1999). Filamentous bacteriophage as a versatile method for aligning RNA, DNA, and proteins for measurement of NMR dipolar coupling interactions. Methods Enzymol. 317, 220-240.

66. Tolman, J. R. \& Prestegard, J. H. (1996). Measurement of amide ${ }^{15} \mathrm{~N}-{ }^{1} \mathrm{H}$ one-bond couplings in proteins using accordion heteronuclear-shift-correlation experiments. J. Magn. Reson. 112, 269-274.

67. Tian, F., Bolon, P. \& Prestegard, J. (1999). Intensitybased measurement of homonuclear residual dipolar couplings from CT-COSY. J. Am. Chem. Soc. 121, 7712-7713.

68. Berman, H. M., Westbrook, J., Feng, Z., Gilliland, G., Bhat, T. N., Weissi, H., Shindyalov, I. N. \& Bourne, P. E. (2000). The Protein Data Bank. Nucl. Acids Res. 28, 235-242.

69. Marion, D., Driscoll, P. C., Kay, L. E., Wingfield, P. T., Bax, A., Gronenborn, A. M. \& Clore, G. M. (1989). Overcoming the overlap problem in the assignment of ${ }^{1} \mathrm{H}$ NMR spectra of larger proteins by use of three-dimensional heteronuclear ${ }^{1} \mathrm{H}-{ }^{15} \mathrm{~N}$ Hartmann-Hahn-multiple quantum coherence and nuclear Overhauser-multiple quantum coherence spectroscopy: application to interleukin $1 \beta$. Biochemistry, 28, 6150-6156.

70. Grzesiek, S. \& Bax, A. (1992). Correlating backbone amide and side chain resonances in larger proteins by multiple relayed triple resonance NMR. J. Am. Chem. Soc. 114, 6291-6293.

71. Muhandiram, D. R. \& Kay, L. E. (1994). Gradientenhanced triple-resonance three-dimensional NMR experiments with improved sensitivity. J. Magn. Reson. 103, 203-216.

72. Wittekind, M. \& Mueller, L. (1993). HNCACB, a high-sensitivity 3D NMR experiment to correlate amide-proton and nitrogen resonances with the alpha and beta-carbon resonances in proteins. J. Magn. Reson. 101B, 214-217.

73. Ikura, M., Kay, L. E. \& Bax, A. (1990). A novel approach for sequential assignment of ${ }^{1} \mathrm{H},{ }^{13} \mathrm{C}$, and ${ }^{15} \mathrm{~N}$ spectra of larger proteins: heteronuclear tripleresonance three-dimensional NMR spectroscopy. Application to calmodulin. Biochemistry, 29, 46594667.

Edited by P. Wright

(Received 25 July 2000; received in revised form 27 September 2000; accepted 29 September 2000)

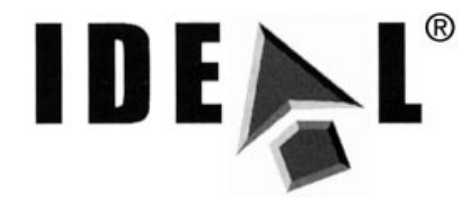

$$
\text { http://www.academicpress.com/jmb }
$$

Supplementary material for this paper is available on IDEAL. 